\title{
GIST Gástrico - Experiência do INCA
}

\author{
Gastric Stromal Tumors- the Experience of the Brazilian National Cancer Institute
}

\author{
Marcus Valadão, Eduardo Linhares, ${ }^{2}$ Leonaldson Castro, ${ }^{3}$ Carlos Eduardo Pinto, ${ }^{3}$ Rodrigo Lugão, ${ }^{4}$ Cláudio Quadros, ${ }^{5}$ Ivanir Martins ${ }^{6}$
}

\section{Resumo}

Introdução: Tumor estromal gastrointestinal (GIST) é uma doença relativamente rara e a cirurgia se constitui no tratamento principal deste tumor. Existem apenas poucos centros com experiência no tratamento desta neoplasia. 0 objetivo do trabalho é analisar os resultados do tratamento cirúrgico dos pacientes portadores de GIST gástrico operados em uma mesma instituição.

M étodos: Estudo retrospectivo baseado na análise de 20 pacientes com diagnóstico confirmado de GIST gástrico operados no Instituto N acional do Câncer (INCA- Rio de Janeiro) entre 1986 e 2000. Todos os dados foram revisados, enfocando-se as características histopatológicas (localização, número de mitoses e tamanho do tumor), as características dos pacientes (idade, sexo e apresentação clínica) e os resultados cirúrgicos (tipos de cirurgia, morbidade, mortalidade e sobrevida de acordo com a classificação proposta por Shiu).

Resultados: 0 nze pacientes eram do sexo masculino e 9 do sexo feminino. A mediana de idade foi de 57 anos. 0 tamanho médio do tumor foi de $14,7 \mathrm{~cm}$. Setenta por cento dos pacientes tinham tumor de alto grau e $65 \%$ dos tumores se localizavam na porção proximal do estômago. Gastrectomia subtotal foi realizada em $50 \%$ dos casos, gastrectomia total em $35 \%$ e gastrectomia atípica em $10 \%$. Ressecção gástrica isolada foi realizada em seis casos (grupo 1), ressecção de dois órgãos foi feita em cinco casos (grupo 2) e ressecção de mais de dois órgãos em oito casos (grupo 3). A morbidade operatória global foi de 35\%. 0 grupo 1 não teve morbidade, o grupo 2 teve morbidade de $20 \%$ e o grupo 3, de $70 \%$. A mortalidade operatória foi de $10 \%$ (2 pacientes). Dez por cento dos pacientes foram classificados como estádio 0,25\% como estádio I e 65\%, estádio II de acordo com a classificação de Shiu. A sobrevida em 5 anos para o estádio 0 foi de 100\% enquanto que 0 estádio II apresentou sobrevida nula em 5 anos.

Conclusão: A alta morbi-mortalidade desta casuística é devido à presença de doença avançada ao diagnóstico, havendo necessidade de ressecções multiorgânicas a fim de se obter cirurgia R0. A cirurgia é o tratamento principal, porém isolada é insuficiente para se atingir sobrevida longa em doença avançada.

Palavras-chave: tumor estromal gastrointestinal; GIST gástrico; neoplasias gástricas; gastrectomia; mortalidade; morbidade.

\footnotetext{
${ }^{1}$ Ex-Residente de cirurgia oncológica da Seção de Cirurgia Abdomino-Pélvica- IN CA.

${ }^{2}$ Chefe da Seção de Cirurgia Abdomino-Pélvica- IN CA, M estre e D outor em Cirurgia Abdominal.

${ }^{3}$ M estre e D outor em Cirurgia Abdominal; Staff da Seção de Cirurgia Abdomino-Pélvica- IN CA.

${ }^{4}$ Ex-Residente de cirurgia oncológica da Seção de Cirurgia Abdomino-Pélvica- IN CA.

${ }^{5}$ Ex-Residente de cirurgia oncológica da Seção de Cirurgia Abdomino-Pélvica- IN CA.

${ }^{6}$ M édica do Serviço de Anatomia Patológica- IN CA.

Endereço para correspondência: email: eduardol@inca.gov.br ou abdomehc@inca.gov.br
} 


\begin{abstract}
Background: Gastrointestinal stromal tumors (GIST) is a relative rare disease and surgery is the cornerstone in the treatment of these tumors. There are few medical centers with experience in the treatment of this neoplasm. The objective of this article is to analyze the results of the surgical treatment of patients with gastric GIST operated on at a particular medical facility.

Methods Retrospective analysis of 20 patients with proven diagnosis of gastric GIST who had been operated at the Brazilian N ational C ancer Institute (IN CA- Rio de Janeiro) from 1986 to 2000. All data were reviewed and focus was given on the histopathology features (localization, number of mitoses and size), patients features (age, gender and clinical presentation), and the surgical outcomes (types of surgery, morbidity, mortality, and survival according to Shiu classification).
\end{abstract}

Results: Eleven patients were males and 9 were females. The median age was 57 years. The median size of the tumor was $14,7 \mathrm{~cm}$. Seventy percent of the patients had high-grade tumors, and $65 \%$ had tumors localized at the proximal stomach. Fifty percent were submitted to subtotal gastrectomy, 35\% to total gastrectomy, and $10 \%$ to atypical gastrectomy. Gastric resection alone was performed in 6 cases (group 1), two organs resection was performed in 5 cases (group 2), and resection of more than two organs was performed in 8 cases (group 3). The overall operative morbidity was 35\%. Group 1 had no morbidity, group 2 had 20\% morbidity and group 3,70\%. The operative mortality was $10 \%$ ( 2 patients). Ten percent of the patients were classified as stage $0,25 \%$ were stage I and $65 \%$ were stage II, according to the Shiu classification. The 5 -year survival of stage 0 was $100 \%$, and there was no 5-year survival in the stage II group.

Conclusion: The high morbidity and mortality reported in this article are due to advanced disease at diagnosis, requiring multiorgan resections in order to reach $\mathrm{RO}$ surgery. Surgery is the main treatment, but alone it is insufficient to achieve long-term survival in advanced disease.

Key words gastrointestinal stromal tumors; gastric GIST; stomach neoplasms; gastrectomy; morbidity; mortality.

\section{INTRODUÇÃO}

Embora relativamente raros, os tumores estromais do trato gastrointestinal (GIST, sigla em inglês para gas trointestinal stromal tumors) compreendem a maioria dos tumores mesenquimais do trato digestivo ${ }^{1}$ e constituem $5 \%$ de todos os sarcomas. ${ }^{2}$

O s GIST são derivados das células intersticiais de Cajal, localizadas ao nível do plexo mioentérico, entre a camada muscular longitudinal e a circular do trato gastrointestinal. As células de $C$ ajal são responsáveis pela motilidade intestinal, ${ }^{3}$ sendo chamadas de células marcapasso do trato gastrointestinal. Essas células apresentam características imunofenotípicas e ultra-estruturais tanto de músculo liso quanto de diferenciação neural, e expressam o receptor Kit (CD 117), semelhante ao tumor estromal gastrointestinal (GIST).,5

0 Kit éum receptor tirosina quinase transmembrana, responsável por várias funções celulares, dentre as quais proliferação, adesão, apoptose e diferenciação celular. ${ }^{6}$ No GIST, a mutação no gene Kit leva a uma ativação constitutiva na proteína Kit, causando um estímulo sem oposição para proliferação celular.

O s GIST podem se originar em qualquer local do trato gastrointestinal, do esôfago ao ânus. Em relação à distribuição, $50 \%$ a $60 \%$ das lesões são provenientes do estômago, $20 \%$ a $30 \%$ do intestino delgado, $10 \%$ do intestino grosso, $5 \%$ do esôfago e $5 \%$ de outros locais da cavidade abdominal. ${ }^{7}$

0 tratamento padrão para pacientes com GIST não metastático é a ressecção cirúrgica completa da lesão, pois oferece a maior chance de cura. 0 tratamento com imatinib (ST I571) é utilizado para doença metastática ou irressecável.

0 objetivo do estudo é analisar os resultados do tratamento cirúrgico de 20 pacientes portadores de G IST gástrico, operados em uma mesma instituição.

\section{PACIENTES E MÉTODOS}

Foram analisados retrospectivamente, através da revisão de prontuários, 20 pacientes portadores de G IST gástrico operados no Instituto $\mathrm{N}$ acional do Câncer (IN CA), no período de 1986 a 2000.

Todos os blocos de parafina e lâminas foram revisados e submetidos a estudo imunoistoquímico. 0 diagnóstico de GIST foi confirmado pela presença do c-Kit positivo à imunoistoquímica.

Algumas características relacionadas ao tumor, como tamanho, localização e número de mitoses, foram consideradas para efeito de análise. Tomamos como padrão 0 número de mitoses por 50 campos de grande 
aumento (CGA), estabelecendo a seguinte classificação: alto grau (mais de 5 mitoses por 50 CGA) e baixo grau (menor ou igual a 5 mitoses por 50 CGA).

$D$ ados demográficos tais como distribuição entre os sexos e idade mediana ao diagnóstico, informações relativas à apresentação clínica, diagnóstico por endoscopia, tipo decirurgia realizada e número de órgãos ressecados por cirurgia foram igualmente considerados em nossa análise.

Todos os pacientes foram submetidos à linfadenectomia D 1 (segundo a classificação da Japanese Gastric Cancer Association ${ }^{8}$ ), tendo a gastrectomia obedecido ao princípio de $2 \mathrm{~cm}$ de margem.

Analisamos a morbidade e a mortalidade, estratificando a morbidade por tipo de cirurgia realizada.

U tilizamos o estadiamento descrito por Shiu e cols., ${ }^{9}$ que consiste na divisão em grupos baseado na presença de três fatores prognósticos desfavoráveis: tumor maior que $5 \mathrm{~cm}$, alto grau de diferenciação e invasão de órgãos adjacentes. No estádio 0 não há nenhum dos três fatores desfavoráveis. N o estádio I está presente apenas 1 fator prognóstico desfavorável, e no II, estão presentes dois ou três fatores prognósticos. A sobrevida foi estimada pelo método de Kaplan-M eier e a diferença das curvas de sobrevida foi analisada pelo teste de log-rank.

\section{RESULTADOS}

Em relação aos dados demográficos, 11 pacientes eram do sexo masculino e 9 do sexo feminino. A idade mediana foi de 57 anos, variando entre 34 e 81 anos (Tabela 1).

Q uatorze pacientes apresentavam tumores de alto grau e 6 pacientes, de baixo grau (Tabela 1). 0 tamanho médio dos tumores foi de $14,7 \mathrm{~cm}$ e apenas dois pacientes tinham tumores menores que $5 \mathrm{~cm}$ (Tabela 1). A localização mais freqüente foi no terço proximal do estômago, ocorrendo em $65 \%$. No terço distal ocorreu em $25 \%$ e em $10 \%$ a localização não pode ser definida devido à grande extensão da lesão (Tabela 1).

Q uanto à apresentação clínica, os sintomas mais comuns foram dor (presente em $70 \%$ da casuística) e massa palpável (presente em 70\%), seguidos de hemorragia digestiva (50\%) e perda de peso (15\%).

A endoscopia digestiva alta foi realizada em $80 \%$ da casuística e apenas $18 \%$ dos casos tiveram o diagnóstico de GIST no pré-operatório.
Tabela 1- Características dos pacientes

\begin{tabular}{|c|c|}
\hline \multicolumn{2}{|c|}{ Características dos pacientes } \\
\hline \multicolumn{2}{|l|}{ Sexo } \\
\hline Masculino & 11 casos \\
\hline Feminino & 9 casos \\
\hline Idade mediana & 57 anos \\
\hline Tamanho do tumor (média) & $14,7 \mathrm{~cm}$ \\
\hline \multicolumn{2}{|l|}{ Grau de diferenciação } \\
\hline Alto grau (>5 mit por 50CGA) & $14 \operatorname{casos}(70 \%)$ \\
\hline Baixo grau $(<=5$ mit por 50CGA) & $6 \operatorname{casos}(30 \%)$ \\
\hline \multicolumn{2}{|l|}{ Localização } \\
\hline Estômago proximal & 13 casos $(65 \%)$ \\
\hline Estômago distal & 5 casos $(25 \%)$ \\
\hline Não definido & $2 \operatorname{casos}(10 \%)$ \\
\hline
\end{tabular}

D as cirurgias realizadas, $50 \%$ foram gastrectomias subtotais, 35\% foram gastrectomias totais, $10 \%$ de gastrectomias atípicas e em 5\% (1 caso) a lesão foi irressecável. Todas as ressecções tiveram intuito curativo, correspondendo a cirurgias R0.

Durante a laparotomia, quatro pacientes apresentavam metástase hepática e dois pacientes tinham implante peritoneal (todas as lesões foram ressecadas). H avia invasão de cólon em dois pacientes e de pâncreas em outros dois.

As cirurgias foram estratificadas em três grupos: cirurgia com ressecção apenas do estômago (6 casos), cirurgia com ressecção de dois órgãos (5 casos) e cirurgia com ressecção de mais de dois órgãos (8 casos).

0 s órgãos mais comumente ressecados foram o baço e o fígado (metastasectomias) (Tabela2).

Tabela 2 - Órgãos ressecados

\begin{tabular}{l|c}
\multicolumn{1}{c|}{ Órgãos ressecados } & $N^{\circ}$ de pacientes \\
\hline Baço & 6 \\
\hline Fígado & 3 \\
\hline Pâncreas & 2 \\
\hline Cólon & 2 \\
\hline Vesícula & 2 \\
\hline Esôfago & 1 \\
\hline Útero & 1 \\
\hline
\end{tabular}

Foram ressecados 195 linfonodos, correspondendo a uma média de 11 linfonodos por ressecção e todos foram negativos para malignidade.

A morbidade operatória foi de $35 \%$, sendo a infecção do sítio cirúrgico a complicação mais freqüente (Tabela 3). Q uando se estratifica a morbidade operatória por grupos de ressecção, observa-se que o grupo apenas com ressecção do estômago não apresenta morbidade, ao passo que o grupo com ressecção de dois órgãos tem morbidade de $20 \%$, e o grupo com ressecção de mais de dois órgãos apresenta morbidade de $70 \%$. 
A mortalidade operatória foi de 10\% (2 casos), sendo um caso relacionado a distúrbio de coagulação e sangramento no pós-operatório, e o outro devido à sepse abdominal em conseqüência de fístula anastomótica.

Em relação à distribuição por estadiamento de Shiu, observou-se que $10 \%$ dos pacientes eram estádio 0, 25\% estádio I e $65 \%$ estádio II.

Tabela 3 - Morbidade operatória

\begin{tabular}{l|c}
\multicolumn{1}{c|}{ Complicações } & $N^{\circ}$ de pacientes \\
\hline Infecção do sítio cirúrgico & 3 casos \\
\hline Infecção respiratória & 1 caso \\
\hline Infecção urinária & 1 caso \\
\hline Fístula & 1 caso \\
\hline Sangramento & 1 caso \\
\hline
\end{tabular}

A análise da sobrevida estratificada por estadiamento segundo Shiu e cols. 9 revelou que a sobrevida em 5 anos (estimada pelo método de Kaplan-M eier) para 0 estádio 0 foi de $100 \%$, para o estádio I foi de $60 \%$ e para 0 estádio II foi nula (Figura 1). H ouve diferença estatisticamente significativa entre os estádios quando utilizado o teste de log-rank $(p<0,01)$.

Figura 1- Tempo de sobrevida conforme o estadiamento de Shiu

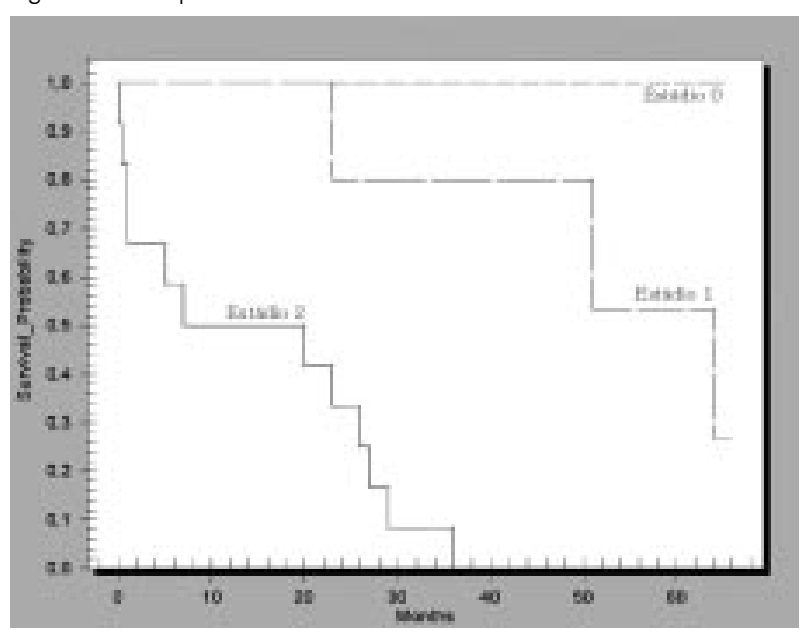

\section{DISCUSSÃO}

N ossos dados corroboram os achados da literatura no que diz respeito aos dados demográficos. O s GIST ocorrem predominantemente entre 40 e 80 anos, sendo a idade média ao diagnóstico em torno dos 60 anos. $^{2} \mathrm{~A}$ incidência entre os sexos é praticamente semelhante. ${ }^{10}$

A apresentação clínica dos pacientes portadores de GIST é variável e se correlaciona principalmente com 0 tamanho da lesão. Lesões pequenas são geralmente achadas incidentalmente à laparotomia para tratamento de outras condições clínicas. Lesões maiores podem ser detectadas por achado radiológico incidental. Assim como outros sarcomas, os GIST tendem, inicialmente, a deslocar estruturas adjacentes sem invadí-las. Conseqüentemente, o tumor pode se manter sem a presença de sintomas até se tornar grande 0 bastante para ser notado como massa palpável e causar desconforto abdominal ( $70 \%$ em nossa casuística). Além disso, a lesão pode erodir para dentro do trato gastrointestinal e causar hemorragia importante $(50 \%$ em nossa casuística).

0 diagnóstico pré-operatório por endoscopia não é freqüente, pois o aspecto radiológico das lesões muito grandes se assemelha com sarcomas retroperitoneais e não sinaliza ao médico a possibilidade de o tumor se originar no próprio trato digestivo. Além disso, as lesões que erodem o estômago apresentam extensa área de necrose, tornando a biópsia por endoscopia insuficiente para o diagnóstico.

0 tratamento padrão para pacientes com GIST não metastático é a ressecção cirúrgica completa da lesão, sendo necessária uma técnica cirúrgica meticulosa, visando prevenir a rotura tumoral durante a ressecção, 0 que teria impacto adverso no prognóstico. ${ }^{11,12,13} \mathrm{~A}$ cápsula tumoral se rompe facilmente e pode resultar em sangramento.

Quando possível, uma ressecção marginal do estômago (com margens de 1 a $2 \mathrm{~cm}$ checadas por congelação) se constitui em um tratamento adequado, não havendo benefício aparente de ressecção alargada de tecidos não envolvidos. ${ }^{9}$

N ossa casuística não evidenciou metástase nodal, evento de natureza rara, ${ }^{2}$ corroborando a posição dos autores quanto à dispensa da linfadenectomia.

A morbimortalidade operatória elevada em nossa casuística, obtida a partir da coleta rigorosa de dados, revela a presença de doença avançada ao diagnóstico (tamanho médio do tumor de $14,7 \mathrm{~cm}, 65 \%$ da casuística em estádio II de Shiu) e a necessidade de ressecções multiorgânicas para se obter cirurgia R0 (sem doença residual).

Em nossa casuística, a sobrevida dos pacientes com doença avançada foi ruim (nula em 5 anos para os pacientes em estádio II de Shiu). A experiência do $\mathrm{Me}$ morial Sloan-K attering Cancer C enter, com 200 pacientes com GIST tratados e seguidos prospectivamente, evidenciou que 80 deles, sem doença metastática, foram submetidos à ressecção cirúrgica completa, tendo sobrevida em 5 anos de $54 \% .^{2}$ A sobrevida média dos pacientes com doença metastática foi de 19 meses, e com doença recidivada, de 12 meses. $^{2}$

A conduta padrão após a ressecção completa do tumor tem sido a observação. Isso, em parte, reflete 0 
não benefício da radioterapia e da quimioterapia convencional como tratamento adjuvante ou mesmo no tratamento de doença metastática.

M uitos pacientes apresentam recidiva apesar da ressecção cirúrgica completa do tumor primário. $\mathrm{Na}$ experiência do MD Anderson Cancer Center, somente $10 \%$ dos pacientes estavam livres de doença após longo acompanhamento. ${ }^{11} 0$ padrão inicial de recidiva envolve, predominantemente, o peritôneo e o fígado. A sobrevida média após o resgate cirúrgico da recidiva é de 15 meses. $^{14}$

A recidiva isolada para a cavidade peritoneal pode ser tratada por cirurgia, porém todos os pacientes irão desenvolver recidivas subseqüentes, apesar da ressecção macroscópica completa das lesões. A doença metastática hepática geralmente se apresenta como doença difusa intra-hepática, impedindo a ressecção cirúrgica. Alguns tipos de tratamento foram utilizados para controlar a doença metastática, como a quimioterapia sistêmica ou intraperitoneal e a embolização da artéria hepática, porém não se constatou benefício.

A descoberta do STI571 (imatinib) revolucionou 0 tratamento do câncer, por ser a primeira terapia a agir especificamente na alteração molecular responsável pela etiologia da doença. 0 conhecimento de mutações (com ganho de função do gene $K$ it) na gênese e progressão dosG IST proporcionou o desenvolvimento deuma droga com alvo molecular definido, que interfere na atividade tirosina quinase dos receptores $\mathrm{Kit} .{ }^{15}$ Inicialmente utilizada no tratamento da leucemia mielóide crônica com grande sucesso, o inibidor da atividade da proteína quinase ST I571 (imatinib) começou a ser utilizada no tratamento dos GIST.

Estudos fase I e II evidenciaram a eficácia do imatinib no tratamento de pacientes portadores de GIST metastático ou irressecável. ${ }^{16,17} \mathrm{H}$ á estudos fase III em andamento nos Estados U nidos.

Recentemente, o imatinib passou a ser considerado para terapia adjuvante após ressecção completa do tumor primário. 0 esperado é que o risco de recidiva após ressecção cirúrgica isolada seja alto devido à doença microscópica residual oculta, impossível de prevenir ou tratar por quimioterapia convencional. Supõe-se que 0 tratamento adjuvante após ressecção completa do GIST com 0 imatinib pode prevenir ou retardar a recidiva e conseqüentemente prolongar a sobrevida.

\section{CONCLUSÃO}

0 conhecimento da biologia tumoral e da história natural desta patologia está a cada dia sendo desvendada a partir da compilação cada vez maior de publicações sobre o tema. A difusão do conhecimento tem sido importante na medida em que novos casos são identificados, possibilitando a formação de uma casuística expressiva.

Muitas questões ainda precisam ser respondidas, porém, à luz do conhecimento atual, sabe-se que a ressecção cirúrgica é o principal tratamento do GIST e que a cirurgia isolada é um tratamento insuficiente para lesões avançadas. É necessária a realização de estudos que respondam qual o possível benefício do tratamento adjuvante em pacientes portadores de GIST de mau prognóstico.

\section{REFERÊNCIAS BIBLIOGRÁFICAS}

1. Graadt JF, Van Velthuysen M LF, H ogendoorn PCW. The histopathological differential diagnosis of gastrointestinal stromal tumors. J Clin Pathol 2001;54:96-102.

2. D eM atteo RP, LewisJJ, Leung D, M udan SS, Woodruff JM , Brennan M F. Two hundred gastrointestinal stromal tumors: recurrence patternsand prognostic factorsfor survival. Ann Surg 2000;231:51-8.

3. Isozaki $\mathrm{K}, \mathrm{H}$ irotaS, $\mathrm{N}$ akamaA. D isturbed intestinal move ment, bile reflux to the stomach, and deficiency of c-K it expressing cells in W s/W smutant rats. G astroenterology 1995;109:456-64.

4. Kindblom LG, Remotti HE, Aldenborg F, M eis-Kindblom JM . Gastrointestinal pacemaker cell tumor (GIPACT): gastrointestenal stromal tumors show phenotypic characteristics of the intestinal cells of Cajal. Am J Pathol 1998;152:1259-69.

5. Li A, N ordberg M L, H errera GA. G astrointestinal stromal tumors: current concepts and controversies. Pathol $C$ ase Rev 2002;7:226-33.

6. H uizinga JD, Thuneberg $L, K$ luppel $M, M$ alysz $J$, $M$ ikkelsen H B, Bernstein A. W/K it generequired for intestinal cells of $C$ ajal and for intestinal pacemaker activity. N ature 1995;373:347-9.

7. Saund M, D emetri G, Asshley S. G astrointestinal stromal tumors (GIST). C urr O pin G astrointerol 2004;20:89-94.

8. KodamaY, Sugimachi K, Soejima K. Evaluation of extensivelymph nodedissection for carcinoma of the stomach. World J Surg 1981;5:242.

9. Shiu M H , Farr G H , Papachristou D N , H adju SI. M yosarcomas of the stomach: natural history, prognostic factors and management. Cancer 1982;49:177-87.

10. Chan JKC. M esenchymal tumors of the gastrointestinal tract: a paradisefor acronyms (ST U M P, GIST, GANT, and now GIPACT ). Implication of c-K it in genesis, and yet another of theemerging roles of the intersticial cell of $C$ ajal in the pathogenesis of gastrointestinal diseases? Adv Anat Pathol 1999;6:19-40.

11. N gEH , Pollock RE, M unsell M F, Atikson EN , Romsdahl $M M$. Prognostic factorsinfluencing survival in gastrointes tinal leiomyosarcomas: implications for surgical manage- 
ment and staging. Ann Surg 1992;215:68-77.

12. Connolly EM, Graffney E, R eynoldsJV. G astrointestinal stromal tumors. BrJ Surg 2003;90:1178-86.

13. Yan $\mathrm{H}, \mathrm{M}$ archettini $\mathrm{P}, \mathrm{Ach}$ erman $\mathrm{YI}$, G ething $\mathrm{SA}$, Brun $\mathrm{E}$, Sugarbaker PH . Prognostic assessment of gastrointestinal stromal tumor. Am J Clin O ncol 2003;26:221-8.

14. M udan SS, Conlon KC, Woodruff J, Lewis J, Brennan M F. Salvagesurgery in recurrent gastrointestinal sarcoma: prognostic factors to guide patient selection. Cancer 2000;88:66-74.

15. D eM atteo RP, M aki RG , Antonescu C, Brennan M F. Tar- geted molecular therapy for cancer: the application of ST I571 to gastrointestinal stromal tumor. Curr Probl Surg 2003;40(3):133-93.

16. Van 0 osterom AT, Judson I, Verweij J, Stroobants S, di Paola ED, D imitrijevic $S$, et al. Safety and efficacy of imatinib (ST I 571) in metastatic gastrointestinal stromal tumors: a phasel study. Lancet 2001;358:1421-3.

17.D emetri $G D$, Von $M$ ehren $M$, BlankeCD, Van den Abbeele $A D$, Eisenberg $B$, Roberts PJ, et al. Efficacy and safety of imatinib mesylatein advanced gastrointestinal stromal tumors. N Engl J Med 2002;347:472-80. 